\title{
Influence of Hydroxypropyl Methylcellulose Molecular Weight Grade on Water Uptake, Erosion and Drug Release Properties of Diclofenac Sodium Matrix Tablets
}

\author{
Jafar Akbari ${ }^{1,2}$, Reza Enayatifard ${ }^{1}$, Majid Saeedi ${ }^{1,2}$ and Massoud Saghafi ${ }^{1}$ \\ ${ }^{1}$ Department of Pharmaceutics, Faculty of Pharmacy, ${ }^{2}$ Pharmaceutical Sciences Research Center, Mazandaran \\ University of Medical Sciences, Sari, Iran
}

\begin{abstract}
Purpose: To comparatively evaluate the effect of two hydroxylpropyl methylcellulose (HPMC) molecular weight grades (K4M and K15M) on drug release from diclofenac sodium matrix tablets.

Methods: Tablets containing diclofenac sodium were prepared by direct compression method at various drug/HPMC ratios and evaluated in vitro for their water uptake, erosion and dissolution characteristics over a period of $8 \mathrm{~h}$. Their release data were analyzed according to various release kinetic models.

Results: The release rate of diclofenac decreased with increase in polymer content and was dependent on the HPMC type used, with the lower release rate observed in formulations containing the higher molecular weight grade HPMC K15M. Formulations containing the higher molecular weight HPMC (F4, F5 and F6) showed higher water uptake than those containing the lower molecular weight polymer (F1, $F 2$ and $F 3)(p<0$. 001). The formulations incorporating the lower molecular weight HPMC K4M (F1, F2 and F3) showed higher erosion than those that contained HPMC K15M (F4, F5 and F6) $(p<0.001)$. Kinetic data based on the release exponent, $n$, in Peppas model, showed that $n$ values were between 0.14 and 0.55 , indicating that drug release from HPMC matrices was predominantly by diffusion.

Conclusion: This study demonstrates that the molecular weight (MW) of HPMC does affect the water uptake and erosion as well as the rate of drug release from of HPMC matrices.
\end{abstract}

Keywords: Matrix, Diclofenac sodium, HPMC, Erosion, Water uptake. 


\section{INTRODUCTION}

The development of controlled or sustained release delivery systems is a tool for optimizing therapeutic effect, by maximizing bioavailability of conventional drugs and reducing their side effects. These systems include matrix tablets, which are considered the easiest strategy for controlled release systems. Compressed hydrophilic matrices are commonly used as oral drug delivery systems and are increasingly being investigated for controlled release applications because of their good compatibility [1].

Hydroxypropyl methylcellulose (HPMC) is probably the most important hydrophilic carrier material used for the preparation of oral controlled drug delivery systems. One of its most important characteristics is high swellability, which has a significant effect on the release kinetics of an incorporated drug [2]. Cellulose ethers, especially HPMC, are frequently used as the basis for sustained release hydrophilic matrix tablets [3].

The overall drug release mechanism of HPMC-based pharmaceutical devices strongly depends on the design (composition and geometry) of the particular delivery system. On imbibing water, HPMC swells, resulting in dramatic changes in polymer and drug concentrations, and increased dimensions of the dosage system. Upon contact with water, the incorporated drug dissolves and diffuses out of the device. Depending on the chain length and degree of substitution of the HPMC type used, the polymer itself dissolves more or less rapidly. Diffusion, water uptake and erosion are the most important rate controlling mechanisms of commercially available controlled release products [2].

Monolithic devices or matrices represent a substantial proportion of the drug delivery systems. For oral administration, they are commonly manufactured as tablets by compaction of microparticulate powders. Generally, their release rate modulation is achieved using different types of polymers, with varying swellability and inertness [1]. The varying conditions along the gastrointestinal tract can potentially affect tablet erosion for a gel matrix tablet, thereby altering drug release rate [4]. Many models for drug release from water uptake and dissolving polymer matrices can be found in the literature [1].

The objective of the present investigations was to comparatively evaluate two HPMC molecular grades - HPMC K4M and HPMC $\mathrm{K} 15 \mathrm{M}$ - for their water uptake and erosion properties in diclofenac sodium matrix tablets as well as drug release characteristics, and thus elucidate the kinetics and mechanism of drug release from the matrices.

\section{EXPERIMENTAL}

\section{Materials}

Diclofenac sodium was received as a gift from Roze Darou Company, Tehran, Iran. HPMC K4M and K15M were received, free of charge, from Colorcon, UK. Magnesium stearate, sodium hydroxide, potassium dihydrogen phosphate, and hydrochloric acid were purchased from Merck Co, Germany.

\section{Formulation and preparation of matrix tablets}

Matrix tablets were directly compressed using $9 \mathrm{~mm}$ flat-faced punches on a hydraulic press after blending the ingredients; the formulations were compacted at constant compression pressure $\left(50 \mathrm{KN} / \mathrm{m}^{2}\right)$. The composition of the matrix tablet formulations is given in Table 1.

Table1: Composition of diclofenac matrix tablets

\begin{tabular}{lccc}
\hline Batch & $\begin{array}{c}\text { Drug } \\
(\mathbf{m g})\end{array}$ & $\begin{array}{c}\text { HPMC } \\
\text { K4M (mg) }\end{array}$ & $\begin{array}{c}\text { HPMC } \\
\text { K15M (mg) }\end{array}$ \\
\hline F1 & 50 & 100 & - \\
F2 & 75 & 75 & - \\
F3 & 100 & 50 & - \\
F4 & 50 & - & 100 \\
F5 & 75 & - & 75 \\
F6 & 100 & - & 50 \\
\hline
\end{tabular}

Trop J Pharm Res, October 2011;10(5):536 


\section{Polymer water uptake studies}

The rate of polymer water uptake by the matrix tablets (F1 - F6) was determined by equilibrium weight gain method [5] using a USP/NF dissolution apparatus 1 (Erweka, DT800 Germany) rotating at $100 \mathrm{rpm}$. The basket plus matrix tablets (F1 - F6) were accurately weighed, placed in the basket of the dissolution apparatus and immersed in $900 \mathrm{ml}$ phosphate buffer $(\mathrm{pH}$ 7.4) maintained at $37{ }^{\circ} \mathrm{C}$ in the dissolution vessel. At regular intervals, the pre-weighed basket-matrix system was withdrawn from the dissolution vessel, lightly blotted with tissue paper to remove excess test liquid and weighed. Percent water uptake, was calculated at each time point using Eq 1.

Water uptake $(\%)=\left\{\left(W_{s}-W_{i}\right) / W_{p}\right\} \times 100 \ldots \ldots(1)$

where $W_{s}$ is the weight of the swollen matrix at time $t, W_{i}$ is the initial weight of the matrix, and $W_{p}$ is the weight of the polymer in the matrix.

\section{Matrix erosion studies}

Standard USP/NF dissolution apparatus no.1 (Erweka, DT800, Germany) was used for the evaluation of the matrix tablets (F1 - F6). The matrix tablets were weighed, placed in the basket of the dissolution apparatus, and subjected to dissolution in $500 \mathrm{ml}$ of $0.05 \mathrm{M}$ phosphate buffer ( $\mathrm{pH} 7.4)$ maintained at 37 ${ }^{\circ} \mathrm{C}$ with the basket rotating at $100 \mathrm{rpm}$. At regular intervals, basket-matrix assembly was removed from the dissolution medium, dried to a constant weight in a hot air oven at $50{ }^{\circ} \mathrm{C}$ [5]. Percent matrix erosion $(E)$ at time, $t$, was calculated from Eq 2.

$$
E(\%)=\left\{\left(W_{i}-W_{t}\right) / W_{i}\right\} \times 100
$$

where $\mathrm{Wi}$ is the initial weight of the matrix and $\mathrm{Wt}$ the weight of the matrix subjected to erosion for time, t. Determinations were in triplicate.

\section{In vitro dissolution}

Dissolution tests were performed according to the USP 24 (paddle method) using a dissolution apparatus (Erweka, DT 800, Germany) at a stirring rate of $100 \mathrm{rpm}$. The test was performed on the matrix tablets (F1 F6) in $900 \mathrm{ml}$ of hydrochloric acid (pH 1.2) for 2 h. Samples were withdrawn from the dissolution medium at predetermined time intervals $(0.5,1,1.5$ and $2 \mathrm{~h})$, filtered (Whatman filter paper) and assayed spectrophotometrically at $276 \mathrm{~nm}$ (Spectonic Genesis 2, USA). After $2 \mathrm{~h}$ of the dissolution test, the dissolution medium was immediately changed to phosphate buffer $(\mathrm{pH} 7.4)$ and the test continued. Samples were withdrawn at intervals $(3,4,5,6,7$, and $8 \mathrm{~h})$ and analyzed as stated above. Triplicate determinations were made and the mean \pm standard deviation (SD) recorded.

\section{Analysis of drug release kinetics}

In order to investigate the mode of drug release from the tablets, the release data were subjected to the following mathematical models: zero order (Eq 3), first order (Eq 4), Higuchi (Eq 5) and Korsemeyer-Peppas (Eq $6)$.

$$
\begin{aligned}
& Q=k_{o} t \\
& \ln (100-Q)=\ln Q_{0}-k_{1} t \\
& Q=k_{H} t^{1 / 2} \\
& Q=k_{p} t^{n}
\end{aligned}
$$

where $Q$ is the percent of drug released at time $t$, and $k_{0}, k_{1}$ and $k_{H}$ are the coefficients of the respective equations; $k_{p}$ is a constant incorporating the structure and geometric characteristics of the release device and $n$ is the release exponent indicative of the mechanism of release. When $n$ approximates to 0.5 , a Fickian/diffusion-controlled release is implied; where $0.5<n<1.0$, non-Fickian transport is indicated while $n=1$ suggests zero order (case II transport). When the value of $\mathrm{n}$ approaches 1.0, phenomenologically, one may conclude that the release is approaching zero order [3]. 


\section{Statistical analysis}

One-way ANOVA with Tukey's post hoc test was used to analyze the dissolution data obtained for each batch of formulation in order to compare the rate of drug release from the matrix tablets. The software used was SPSS, version 12 and the confidence limit was set at $95 \%$.

\section{RESULTS}

\section{Water uptake and erosion of matrix}

Water uptake and erosion data are shown in Figs 1 and 2. Water uptake increased with time. The water uptake data may correlate with the molecular weight grade of HPMC, as the formulations containing the higher molecular weight HPMC (F4, F5 and F6) showed higher water uptake than those containing the lower molecular weight polymer (F1, F2 and F3 $(p<0$. 001). Also, increase in the content of polymer in the matrix tablets resulted in water uptake increase $(p<0.001)$.

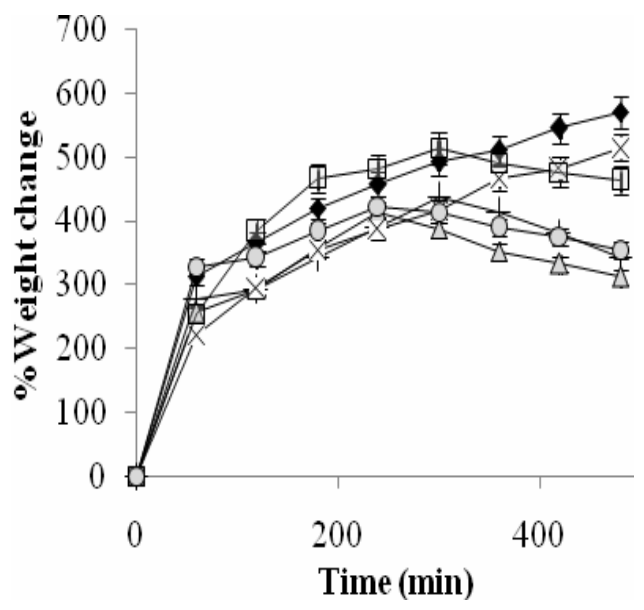

Fig 1: Water uptake of matrix tablets formulations $F_{1}$ $(\times), F_{2}(+), F_{3}(\Delta), F_{4}(\diamond), F_{5}(\square)$, and $F_{6}(\bigcirc)$. (Error bars represent standard deviation)

All the formulations investigated eroded over time. The formulations incorporating the lower molecular weight HPMC K4M (F1, F2 and F3) showed higher erosion than those that contained HPMC K15M (F4, F5 and F6) $(p<0.001)$. Furthermore, increase in the polymer content of the matrix tablets decreased erosion $(p<0.001)$.

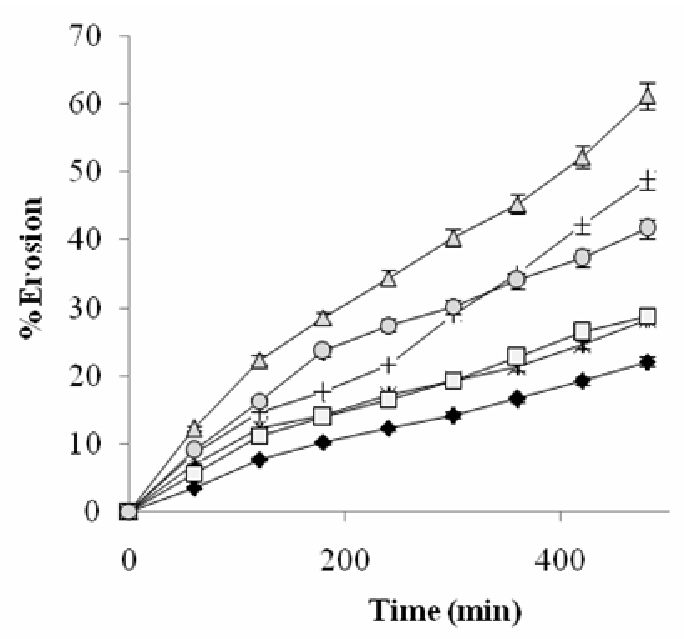

Fig 2: Erosion of matrix tablet formulations $F_{1}(x), F_{2}$ $(+), F_{3}(\Delta), F_{4}(\triangleleft), F_{5}(\square)$, and $F_{6}(\bigcirc)$. (Error bars represent standard deviation)

\section{Drug release from diclofenac matrix tablets}

The results of the dissolution studies, shown in Figs 3, indicate that formulations F1, F2 and F3 (which contain the lower molecular weight HPMC) released 33.5, 42.8 and 56.3 $\%$ of diclofenac, respectively, after $2 \mathrm{~h}$, and $65.6,84.3$ and $99.8 \%$ of drug, respectively, after $8 \mathrm{~h}$. On the other hand, formulations F4, F5 and F6 released 28.3, 39.0 and $46.9 \%$ of diclofenac, respectively, at the end of $2 \mathrm{~h}$, and $60.5,80.0$ and $94.3 \%$ of drug, respectively, after $8 \mathrm{~h}$. The results showed significant differences between $\mathrm{F} 1$ and F4, F2 and F5, F3 and F6 $(p<0.001)$.

Increasing in polymer content resulted in a decrease in release rate $(p<0.001)$ irrespective of the molecular weight of the polymer.

\section{Drug release kinetics}

The results of the analysis of drug release kinetics based on various models are shown in Table 2. It is evident that formulations F1, 
F3, F5 and F6 followed most closely Korsemeyer-Peppas release pattern while formulations $\mathrm{F} 2$ and F4 showed greater approximation to first order and Higuchi models, respectively.

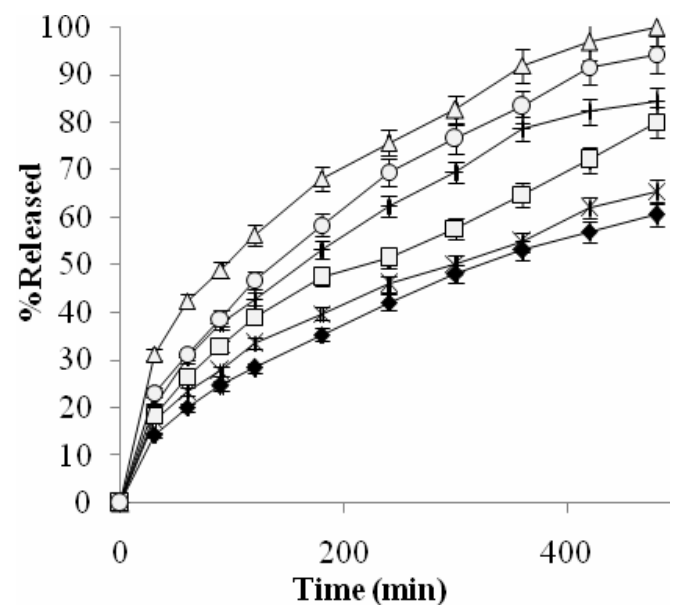

Fig 3: In vitro release of diclofenac sodium from formulations $F_{1}(x), F_{2}(+), F_{3}(\Delta), F_{4}(\bullet), F_{5}(\square)$, and $F_{6}(\bigcirc)$. (Error bars represent standard deviation)

\section{DISCUSSION}

Water uptake of hydrophilic polymeric matrices has been a subject of interest for a number of years. Drug release from a matrix dosage form and its subsequent absorption is dependent on the physicochemical properties of the drug and delivery system, as well as the physiologic environment within the gastrointestinal tract.

\section{Matrix water uptake and erosion}

HPMC is a propylene glycol ether of methylcellulose and hence its physicochemical properties are strongly affected by its methoxy group, hydroxypropyl group content, and molecular weight [2]. In the present study, the matrix tablets containing the higher molecular weight HPMC exhibited higher water uptake. Wan et al has shown that the normalized increase in matrix thickness after 30 min of water uptake for HPMC matrix tablets is higher for higher molecular weight HPMC grades. They attributed this to the larger hydrodynamic volume occupied by higher molecular weight chains when hydrated. As the polymer chains becomes more hydrated and the gel more dilute, the disentanglement concentration may be reached, that is, the critical polymer concentration below which the polymer chains disentangle and detach from a gelled matrix. The polymer then undergoes simultaneous water uptake, dissolution and diffusion into the bulk medium, resulting in the erosion of the polymer [4]. The changes in matrix weight, characteristic of water uptake, started from the onset of the water uptake test and continued until the $8^{\text {th }}$ hour

Table 2: Release kinetic parameters for diclofenac matrix formulations

\begin{tabular}{|c|c|c|c|c|c|c|c|c|c|}
\hline \multirow[t]{2}{*}{$\mathbf{F}^{\star}$} & \multicolumn{2}{|c|}{ Zero order } & \multicolumn{2}{|c|}{ First order } & \multicolumn{2}{|c|}{ Higuchi } & \multicolumn{3}{|c|}{ Korsemeyer-Peppas } \\
\hline & $\begin{array}{c}k_{0} \\
\left(\% h^{-1}\right)\end{array}$ & $R^{2}$ & $\begin{array}{c}k_{1} \\
\left(h^{-1}\right)\end{array}$ & $R^{2}$ & $\begin{array}{c}k_{H} \\
\left(\% h^{-1 / 2}\right)\end{array}$ & $R^{2}$ & $\begin{array}{c}K_{K p} \\
\left(\% h^{-n}\right)\end{array}$ & $n$ & $R^{2}$ \\
\hline $\mathrm{F} 1$ & 0.0014 & 0.9788 & -0.0020 & 0.9961 & 0.0314 & 0.9931 & 0.0640 & 0.51 & 0.9971 \\
\hline $\mathrm{F} 2$ & 0.0011 & 0.9781 & -0.0041 & 0.9951 & 0.0447 & 0.9694 & 0.0510 & 0.41 & 0.9652 \\
\hline F3 & 0.0015 & 0.9586 & -0.0104 & 0.8166 & 0.0443 & 0.9967 & 0.0597 & 0.46 & 0.9990 \\
\hline F4 & 0.0011 & 0.9757 & -0.0017 & 0.9962 & 0.0287 & 0.9991 & 0.0232 & 0.55 & 0.9989 \\
\hline $\begin{array}{l}\text { F5 } \\
\text { F6 }\end{array}$ & $\begin{array}{l}0.0013 \\
0.0018\end{array}$ & $\begin{array}{l}0.9885 \\
0.9814\end{array}$ & $\begin{array}{l}-0.0024 \\
-0.0067\end{array}$ & $\begin{array}{l}0.9876 \\
0.9515\end{array}$ & $\begin{array}{l}0.0352 \\
0.0454\end{array}$ & $\begin{array}{l}0.9951 \\
0.9984\end{array}$ & $\begin{array}{l}0.0370 \\
0.0309\end{array}$ & $\begin{array}{l}0.48 \\
0.55\end{array}$ & $\begin{array}{l}0.9994 \\
0.9991\end{array}$ \\
\hline
\end{tabular}

Note: $F=$ formulation batch; $K_{0}=$ zero order release rate constant; $K_{1}=$ first order release rate constant; $K_{H}=$ Higuchi release rate constant; $K_{k p}=$ Korsmeyer-Peppas release constant; and $n=$ diffusional exponent 
These matrices showed a high ability to swell, and visual observation indicates that at the beginning, a viscous gel mass was created when they came into contact with the liquid.

The investigated polymers eroded over time, with the lower molecular weight HPMC exhibiting the more rapid rate of erosion. No lag time prior to the onset of erosion was observed. Weight loss from the tablets was a function of time and was constantly progressed until the end of 7th hour [7]. An inverse relationship between erosion rate constant and molecular weight has been reported by Reynolds et al [8] while Tahara et al [9] stated that the lower viscosity polymer eroded faster than the higher viscosity one. These findings are consistent with the results of the present work. Thus, the higher molecular weight HPMC polymers had a higher intrinsic water holding capacity and the matrices formed from such polymers were less prone to erosion [6].

\section{Drug release}

The drug release profiles obtained indicated two distinct phases of drug release - an initial burst phase followed by a controlled release phase which is associated with hydration/water uptake of the polymer [10].

Tablets containing HPMC K15M displayed the lower release rate; this polymer is also of higher viscosity grade in view of its greater molecular weight. Thus, the greater degree of entanglement at high molecular weight would reduce the effective molecular diffusion area and hence drug permeation across the matrix gel. This supports the observation of Nellore et al that the higher viscosity gel layers of Methocel K100M matrix provided a more tortuous and resistant barrier to diffusion than Methocel K100LV matrix, resulting in slower release of metoprolol tartrate from the matrix of the former [1].

Drug release from HPMC systems has been reported to be influenced by polymer concentration, drug: polymer ratio, polymer particle size, and the polymer's degree of substitution [11]. The decrease observed in drug release in our study as HPMC content increased, is likely due to increase in the viscosity of the polymer gel leading also to a longer diffusional path. The result would be a decrease in the effective diffusion coefficient of the drug and hence reduction in the drug release rate.

In the present study, it seems that when the matrix tablet was placed in the dissolution medium, initial drug release occurred from the outer surface/layers of the tablets, which constituted 'burst' release. Subsequently, water molecules travelled through long, tortuous channels of the matrix to reach the drug in the deeper layers of the tablet [12]. As erosion gradually reduces the diameter of the tablet, the diffusion path length also decreased [12]. When the penetration of water in the gel matrix exceeds a critical concentration (i.e., the concentration at which the interaction between water and polymer increased, with a consequent reduction of polymer-polymer interactions), the polymer chains begin to separate, thus further exposing the drug. At this stage, the erosion rate increases [12].

In order to achieve the desired release, therefore, the relative rate of hydration of the polymer plays a critical role since the polymer selected must hydrate quickly enough to form a gel layer before the contents of the matrix tablet dissolves. The higher the viscosity of the gel, the more resistant the gel is to dilution or erosion; thus, the viscosity of a gel also is a rate-controlling factor in drug dissolution. If the matrix gel has good durability, soluble drugs may diffuse out of the gel before matrix erosion occurs. Thus, both diffusion and erosion contribute to controlling drug release from a hydrophilic matrix, although one process often plays a predominant role over the other, depending on polymer characteristics [5]. 


\section{Drug release kinetics}

The results of the kinetic analysis of the drug release data indicate the various formulations did not follow a particular model. According to the Noyes-Whitney and Nernst-Brunner drug dissolution models, several factors may influence drug dissolution kinetics. These include the effective surface area of the solid drug, diffusion coefficient of the drug, thickness of the diffusion layer, saturation solubility of the drug, volume of the dissolution medium, and the amount of drug in the solution [10]. It is evident from the present work that swellable matrix tablets are activated by water, and drug release is controlled by the interaction between water, polymer and drug. Thus delivery kinetics depends on the drug gradient in the gel layer and hence, the drug concentration and thickness of the gel layer governed drug flux. The drug release kinetic data show that diffusion played a predominant role in drug release from matrix tablets.

\section{CONCLUSION}

The present study indicates that the molecular weight of HPMC influences the water uptake and erosion of HPMC matrices and that the gel strength of the matrix was important in this regard. Gel strength was, in turn, controlled by the concentration, viscosity and chemical structure of the polymer. Drug release kinetic analysis showed that various formulations followed different kinetic models.

\section{REFERENCES}

1. Escudero JJ, Ferrero C, Castellanos MRJ. Compaction properties, drug release kinetics and fronts movement studies from matrices combining mixtures of swellable and inert polymers: Effect of HPMC of different viscosity grades. Int. J. Pharm., 2008; 361: 61-73.

2. Siepmann J, Peppas NA., Modeling of drug release from delivery systems based on hydroxypropyl methylcellulose (HPMC). Adv Drug Deliv Rev, 2001; 48: 139-157

3. Enayatifard $R$, Saeedi $M$, Akbari J, Haeri Tabatabaee Y., Effect of hydroxypropyl methylcellulose and ethyl cellulose content on release profile and kinetics of diltiazem $\mathrm{HCl}$ from matrices. Trop. J. Pharm. Res., 2009; 8: 425-432.

4. Abrahamsson $B$, Alpsten $M$, Bake B, Larsson $A$, Sjogren J. In vitro and in vivo erosion of two different hydrophilic gel matrix tablets. Eur. J. Pharm. Biopharm., 1998; 46: 69-75.

5. Roy DS, Rohera BD. Comparative evaluation of rate of hydration and matrix erosion of HEC and HPC and study of drug release from their matrices. J. Pharm. Biopharm., 2002; 16: 193199.

6. Kavanagh N, Corrigan OI. Swelling and erosion properties of hydroxypropylmethylcellulose (Hypromellose) matrices-influence of agitation rate and dissolution medium composition. Int. J. Pharm., 2004; 279: 141-152.

7. Vendruscolo CW, Andreazza IF, Ganter JLMS, Ferrero C, Bresolin TMB. Xanthan and galactomannam (from $M$. Scabrella) matrix tablets for oral controlled delivery of theophylline. Int. J. Pharm., 2005; 296: 1-11.

8. Reynolds TD, Gehrke SH, Hussain AS, Shenouda LS. Polymer erosion and drug release characterization of hydroxypropyl methylcellulose matrices. J. Pharm. Sci., 1998; 87: 1115-1123.

9. Tahara $K$, Yamamoto $K$, Nishihata $T$. Overall mechanism behind matrix sustained release (SR) tablets prepared with hydroxypropyl methylcellulose. J. Control. Rel., 1995; 2910: 59-66.

10. Missaghi S, Fassihi R. Release characterization of dimenhydrinate from an erodible and swelling matrix: selection of appropriate dissolution apparatus. Int. J. Pharm., 2005; 293: 35-42.

11. Mourao SC, Silva $C D$, Bresolin TMB, Serra $C H R$, Porta V. Dissolution parameters for sodium diclofenac-containing hypromellose matrix tablet. Int. J. Pharm., 2010; 386: 210-207.

12. Pather SI, Russel I, Syce JA, Neau SH. Sustained release theophylline tablets by direct compression part 1: formulation and in vitro testing. Int. J. Pharm., 1998; 164: 1-10. 